\title{
Définition et conception du non-verbal pour le "psychologue »
}

\author{
Jacques Cosnier
}

\section{OpenEdition}

\section{Journals}

Édition électronique

URL : https://journals.openedition.org/communicationorganisation/2398

DOI : 10.4000/communicationorganisation.2398

ISSN : $1775-3546$

\section{Éditeur}

Presses universitaires de Bordeaux

\section{Édition imprimée}

Date de publication : 1 novembre 2000

ISSN : 1168-5549

Référence électronique

Jacques Cosnier, « Définition et conception du non-verbal pour le "psychologue » », Communication et organisation [En ligne], 18 | 2000, mis en ligne le 21 janvier 2021, consulté le 05 août 2021. URL : http:// journals.openedition.org/communicationorganisation/2398; DOI : https://doi.org/10.4000/ communicationorganisation.2398

Ce document a été généré automatiquement le 5 août 2021 .

(C) Presses universitaires de Bordeaux 


\title{
Définition et conception du non- verbal pour le « psychologue »
}

\author{
Jacques Cosnier
} ce dernier titre que je parlerai essayant d'adopter un point de vue à la fois généraliste et personnel, la difficulté principale venant du fait qu'aujourd'hui il y a certainement plusieurs Psychologies : il est probable qu'un comportementaliste n'aura pas les mêmes conceptions du non-verbal qu'un cognitiviste ou un psychanalyste...

\section{Les termes}

4 Non-Verbal implique une définition de Verbal qui pourrait être la suivante: « le Verbal est tout ce qui peut s'écrire de l'activité discursive ».

5 Un texte écrit (avec un traitement de texte) est donc du Verbal pur, un texte oralisé est du Verbal mis en Voix, et donc sera du verbal combiné avec du Vocal

6 Selon ce point de vue, le Verbal constitue donc le textuel de l'énoncé. Mais cet énoncé, que l'on peut définir comme l'ensemble de tous les événements survenant au cours de l'interaction de face à face du fait des interactants, est «multicanal»: les interlocuteurs s'entendent mais simultanément peuvent aussi se voir, se toucher, se sentir, ou s'efforcer de ne pas le faire, ou de le faire selon des règles...

7 On peut ainsi distinguer : 


\section{Le canal acoustique : « de bouche à oreille »}

8 Par lui passent deux catégories d'informations étroitement liées et complémentaires, mais de nature différente :

- les informations "linguistiques" véhiculées par les paroles. Ces dernières obéissent aux contraintes du code linguistique avec ses règles morpho-syntaxiques (phonologiques, syntaxiques, lexicales) et s'ordonnent en une suite d'énoncés verbaux formant la chaîne syntagmatique du « discours » (ou « texte »).

- les informations «paralinguistiques » liées aux qualités et aux variations vocales (intensité, hauteur, intonation, accent, timbre, tempo...)

9 Parfois ces deux modes sont qualifiés «auditif» ou «verbal» pour le premier, « acoustique » ou « vocal » pour le second, on parlera couramment ainsi de « verbalité » versus « vocatité ».

10 Il faut aussi signaler pour mémoire une troisième catégorie acoustique : les bruits corporels (toux, claquement de langue, rires, soupirs, etc.

\section{Le anal visuel : « de corps à œil »}

Par le canal visuel passent des informations de plusieurs natures

- des informations contextuelles, qui sont fournies les marques spécifiques des interactants : âge, sexe, ethnie, biotype etc... et des marqueurs d'appartenance et/ou de statut social : style vestimentaire, uniforme, insignes, coiffure, tatouages, accents, manières gestuelles et vocales, fournies aussi par des dispositifs scéniques (proxémiques) de l'espace transactionnel.

Ce non-verbal contextuel statique est non événementiel et donc n'est pas constitutif du message énoncé, mais participe au cadrage et aux ancrages référentiels et intervient dans les processus d'implicitation et d'interprétation.

- des informations cotextuelles: la "posturo-mimo-gestualité » (ou "kinésique » essentiellement les mimiques (faciales) et la gestualité corporelle (particulièrement les mouvements des membres supérieurs). Le regard fait partie de ces éléments kinésiques.

\section{Canaux habituellement auxiliaires : « de corps à corps »}

- tactile : le toucher (contact, coup, caresse) ne joue normalement qu'un rôle accessoire dans les interactions sociales de type conversationnel. Peu fréquent dans les cultures Nordiques et Extrême Orientales, plus banal dans les cultures Méditerranéennes (où il participe à la fonction phatique). À noter cependant son importance prépondérante lors des interactions sexuelles, ce qui explique à la fois sa connotation érotique dans certaines cultures, et corollairement son inhibition et sa prohibition, et en tous cas sa formalisation rituelle (poignée de mains, baisers, accolades) dans la plupart, à noter aussi son importance dans les relations de soins.

- thermique : la chaleur du corps d'autrui n'est perçue qu'à distance intime et en tant que phénomène d'évolution lente, elle se situe plutôt dans les paramètres contextuels. 
Chimique : les «phéromones» (molécules chimiques utilisées dans la communication) empruntant deux canaux :

- olfactif: les odeurs naturelles du corps sont dans de nombreuses cultures occidentales réprimées et évitées. Dans les mêmes cultures cependant les parfums et déodorants sont largement commercialisés et appréciés comme marqueurs sociaux et catégoriels, ils participent à ce titre aux informations contextuelles.

14 À noter que l'habituation olfactive est généralement rapide: une odeur est sentie pendant quelques secondes puis n'est plus perçue, ce qui souligne bien son statut de signal contextuel.

- gustatif: la saveur d'autrui n'intervient guère que dans les rapports amoureux, mais pourrait aussi jouer un rôle dans les inducteurs de comportement maternel.

\section{La notion d'énoncé total}

Pour résumer, à partir des éléments précédents, on est amené à distinguer dans les interactions de face à face ordinaires: le contexte constitué par les éléments permanents du cadre et par les éléments permanents (ou à variation lente) liés aux personnes. Le contexte comprend l'ensemble des éléments invariants de la situation où se déroule l'interaction.

L'énoncé total ou " totexte », constitué par la somme de tous les événements survenant au cours de l'interaction du fait de l'activité des interactants.

Le totexte comprend lui-même :

- le texte : événements verbaux (les paroles échangées).

- le cotexte : environnement verbal, vocal et gestuel du texte,

comprenant en synchronie avec ce dernier des :

- événements vocaux (parfois dénommés : «paratexte »)

- événements moteurs : mimiques et gesticulations (« kinésique »)

Les trois principaux constituants de l'énoncé total ou totexte sont donc: la verbalité (que l'on peut écrire) la vocalité (que l'on peut décrire) la kinésique (que l'on peut décrire)

\section{La hiérarchie des constituants du totexte}

On a tendance dans la vie sociale ordinaire à privilégier le texte (l'énoncé verbal) et à considérer les éléments cotextuels (vocaux et kinésiques) comme accessoires ou complémentaires.

Cette attitude est sans doute secondaire au statut particulier donné à la verbalité à la suite de l'invention de l'écriture, mais l'observation des communications totales en face à face offre une autre perspective : les 3 constituants fonctionnent en synergie, peuvent tour à tour être le support principal de l'information, et en permanence contribuent de façon décisive au déroulement de l'interaction, chacun cependant avec des prédispositions spécifiques à assurer telle ou telle fonction. Ainsi les unités significatives de la chaine verbale sont le plus souvent arbitraires (ou «digitales »), tandis que celles de la chaîne kinésique seront plus facilement iconiques (ou " analogiques »). Les premières obéissent à la loi du tout ou rien (non graduelles), sont 
discrètes (discontinues) et indépendantes de la nature des objets référés (arbitraires). Les secondes sont le plus souvent graduables et continues et sont en rapport métonymique ou métaphorique avec les référents. Ainsi la partie rationnelle de la communication, celle qui se conforme le plus facilement aux lois des processus secondaires décrits par les psychanalystes, s'exprime de préférence par le canal verbal, tandis que les affects et les fantasmes inconscients plus liés aux processus primaires, emprunteront préférentiellement les voies corporelles co-textuelles. Soulignons cependant l'indépendance du concept de conventionalité par rapport aux catégories précédentes : un signe arbitraire est toujours conventionnel, mais un signe analogique peut l'être aussi. Ainsi une grande partie de la mimogestualité est liée dans ses formes et dans son usage à la culture et souvent conventionnalisée en dépit de sa nature iconique-analogique, il y a ainsi une manière italienne de gestuer différente de la manière japonaise, nord américaine etc..

D'autre part la répartition intercanale des fonctions de la communication peut varier ainsi que la composition du totexte quand certains canaux sont déficitaires ou inutilisables; tels sont les cas des communications écrites, des communications téléphoniques, des communications entre aveugles, des communications entre sourds.

Dans tous ces cas un canal va devenir prédominant par nécessité et tendra alors à assurer à lui seul la plupart des fonctions ordinairement réparties entre les différents canaux.

\section{L'organisation systémique du totexte : combinaison et non addition des constituants}

L'énoncé total est donc une Gestalt, c'est-à-dire n'est pas simplement la juxtaposition d'éléments verbaux, vocaux et kinésiques. Chaque élément en effet est susceptible de modifier par sa présence le statut et la fonction, et donc la signification, de l'énoncé total et réciproquement. Ainsi un élément gestuel peut renforcer, confirmer la partie verbale, mais aussi la contredire, la remplacer, ou apporter en parallèle une information différente.

En résumé, un message énoncé en face à face résulte de la combinaison de tous les événements verbaux, vocaux et gestuels survenant au cours d'une interaction, et une interaction conversationnelle comprend une combinaison multicanale et plurisémiotique de Verbal et de Non-verbal

\section{Statut du Non-verbal versus le Verbal}

Le Verbal, c'est-à-dire ce système de communication conventionnel (voire arbitraire) propre à l'espèce humaine a joui de tout temps d'un statut privilégié voire ontologique : au début était le Verbe... Ce statut lui est acquis dans la psychologie populaire mais aussi dans la psychologie académique.

En psychologie dynamique on peut dire grossièrement que le verbal (processus secondaire de Freud) est le mode d'expression type de l'activité consciente: le conscient se confond avec le dicible. La représentation de mot est ce qui peut ramener à la conscience la représentation de choses refoulée. 
28 Dans la même ligne on oppose souvent la parole à l'action et les mots aux gestes. De même et presqu'en corollaire les séances de thérapies «non-verbales " comportent souvent un temps de verbalisation. Enfin il est coutumier de dire, comme je l'ai signalé plus haut, que le non-verbal est prédisposé à exprimer l'inconscient: le non-verbal serait moins contrôlé que le verbal et donc plus transparent.

29 Ces opinions sont probablement basées sur le fait que l'expression des émotions spontanées a des impacts directs sur le non-verbal-vocal : modifications du timbre et de la prosodie, et sur le non-verbal gestuel : en particulier mimiques faciales. Et c'est un fait que les états émotionnels intenses sont difficiles à dissimuler.

30 Mais, divers travaux ont aussi montré que les activités verbales et motrices ont des actions régulatrices sur les états émotionnels et donc sur leurs manifestations nonverbales : nous avons décrit cela sous le terme de "phénomène du balancement ».

31 Notons enfin que chacun utilise le verbal et le non-verbal de façon personnalisée : on observe pour reprendre une expression ancienne de Watson des organisations VerboViscero-Motrices variables, certains individus étant très verbalisés, d'autres très motorisés, d'autres à la fois les deux et d'autres enfin s'avérant assez démunis de ces moyens régulateurs.

Voilà donc quelques jalons pour situer cette entité hétérogène qui se caractérise, comme sa dénomination l'indique à juste titre, plus par ce qu'elle n'est pas que par ce qu'elle est, je me propose d'en développer certains aspects dans ma conférence.

\section{AUTEUR}

JACQUES COSNIER

ICAR UMR 5191 